\title{
On the nature of superconductivity in the anisotropic dichalcogenide $\mathrm{NbSe}_{2}\left\{\mathrm{CoCp}_{2}\right\}_{x}$
}

\author{
E-W Scheidt ${ }^{1}$, M Herzinger ${ }^{1}$, A Fischer ${ }^{1}$, D Schmitz ${ }^{1}$, J Reiners ${ }^{1}$, F Mayr $^{2}$, \\ F Loder $^{3}$, M Baenitz $^{4}$ and W Scherer ${ }^{1}$ \\ ${ }^{1}$ CPM, Institut für Physik, Universität Augsburg, 86135 Augsburg, Germany \\ ${ }^{2}$ EPV, EKM, Institut für Physik, Universität Augsburg, 86135 Augsburg, Germany \\ ${ }^{3}$ EPVI, EKM, Institut für Physik, Universität Augsburg, 86135 Augsburg, Germany \\ ${ }^{4}$ Max-Planck-Institut für Chemische Physik fester Stoffe, 01187 Dresden, Germany \\ E-mail: Wolfgang.Scherer@physik.uni-augsburg.de
}

\begin{abstract}
We present a detailed study of the superconducting properties of the weakly pinned, quasi-two-dimensional superconductor $2 \mathrm{H}-\mathrm{NbSe}_{2}$, and its intercalated variant

$\mathrm{NbSe}_{2}\left\{\mathrm{CoCp}_{2}\right\}_{0.26}$. The intercalation of $2 \mathrm{H}-\mathrm{NbSe}_{2}$ with the organometallic donor molecule cobaltocene $\left(\mathrm{CoCp}_{2}\right)$ hardly affects the superconducting properties within the layers. However, the properties perpendicular to the layers change significantly due to the large expansion of the layer spacings of the host lattice in the $c$-direction by a factor of about two. In particular, the superconducting anisotropy factor $\Gamma$ increases from 3.3 in the parent compound $2 \mathrm{H}-\mathrm{NbSe}_{2}$ up to 4.4 in the intercalated species. Therefore, $\mathrm{NbSe}_{2}\left\{\mathrm{CoCp}_{2}\right\}_{0.26}$ is an excellent candidate to analyze how the anisotropy effects the superconducting mechanism in layered dichalcogenides, and to evaluate the various models proposed in the literature to account for the anisotropy in $2 \mathrm{H}-\mathrm{NbSe}_{2}$. While a two-gap model and an anisotropic single-gap model are competing concepts to describe the almost linear $T^{2}$-dependence of $\Delta C / T$ in low-dimensional dichalcogenides, our comparative study suggests that a single-gap model with an anisotropic Fermi-surface is sufficient to capture the $\Delta C / T(T)$ behavior in our samples qualitatively.
\end{abstract}

Keywords: low-dimensionality, superconductors, BCS-theory, dichalcogenide

(Some figures may appear in colour only in the online journal)

\section{Introduction}

Among the numerous studies dealing with the incidence and nature of superconductivity, low-dimensional materials play an increasingly important role as benchmark and model systems [1]. The reason for this is closely connected with the discovery of novel quasi-one or two-dimensional superconductors, such as the rare-earth transition metal carbide $\mathrm{Sc}_{3} \mathrm{CoC}_{4}$ [2,3], or the boride $\mathrm{MgB}_{2}$ and the intercalated, ironbased selenides $\mathrm{Li}_{x} \mathrm{Fe}_{2} \mathrm{Se} 2\left(\mathrm{NH}_{3}\right)_{y}$ [4,5], respectively. Here we report on superconductivity in the new highly anisotropic layered dichalcogenide $\mathrm{NbSe}_{2}\left\{\mathrm{CoCp}_{2}\right\}_{0.26}$, where cobaltocene $\left(\mathrm{CoCp}_{2}=\left(\mathrm{C}_{5} \mathrm{H}_{5}\right)_{2} \mathrm{Co}\right)$ represents an intercalated electronic donor molecule.
Quasi-two-dimensional (2D) intercalation compounds of transition metal dichalcogenide superconductors are typically characterized by highly pronounced anisotropic electronic properties [1]. Despite extensive studies, the superconducting mechanism of this class of compounds is still poorly understood. Among these compounds, $2 \mathrm{H}-\mathrm{NbSe}_{2}$ represents perhaps the most prominent low-dimensional material studied during the past fifty years [6]. After the discovery of the high temperature superconductor $\mathrm{MgB}_{2}$ [4], a benchmark system for multi-band superconductivity [7], $2 \mathrm{H}-\mathrm{NbSe}_{2}$ experienced a renaissance of research activities [8]. In particular, it represents a well-suited candidate for probing the multi-band model in a 2D superconductor, due to the negligible extent of vortex pinning. Furthermore, the critical temperature of 


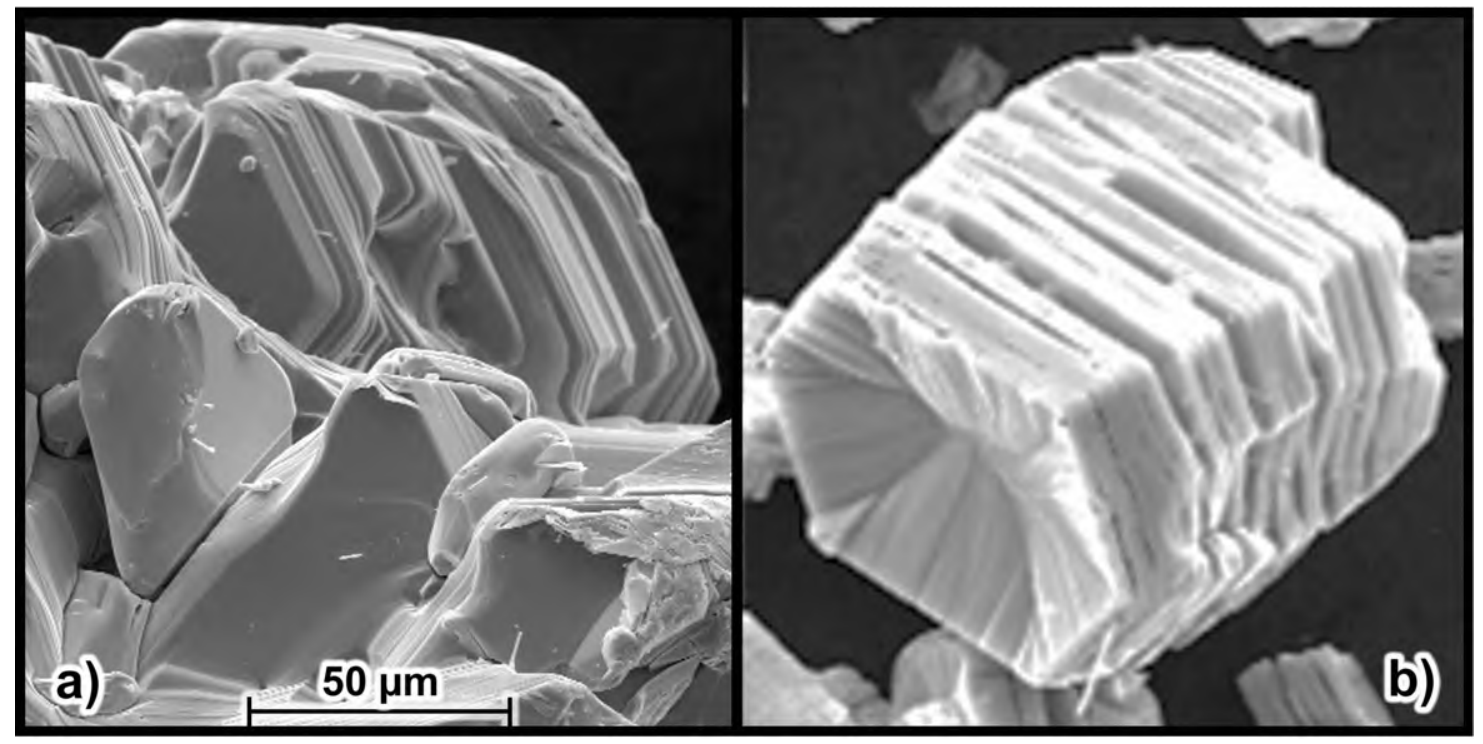

Figure 1. ESEM-micrograph of $(a) 2 \mathrm{H}-\mathrm{NbSe}_{2}$ and $(b) \mathrm{NbSe}_{2}\left\{\mathrm{CoCp}_{2}\right\}_{0.26}$ crystals reflecting the layered structure of these materials.

$T_{\mathrm{c}}=7.2 \mathrm{~K}$ is the highest temperature reported hitherto for dichalcogenid systems [9]. Besides this, $2 \mathrm{H}-\mathrm{NbSe}_{2}$ shows a re-entrant peak effect, as evidenced by resistivity and ACsusceptibility measurements [10]. An additional feature is the presence of a charge density wave (CDW) transition at the Peierls-temperature $T_{\mathrm{CDW}}=33 \mathrm{~K}$ [11]. The reason for the origin and emergence of such a CDW in $\mathrm{NbSe}_{2}$ remains up to now an unresolved question [12].

In order to enhance the anisotropic character we designed the geometrical and electronic structure of $2 \mathrm{H}-\mathrm{NbSe}_{2}$ via the intercalation of high quality $2 \mathrm{H}-\mathrm{NbSe}_{2}$ single crystals with the organometallic donor molecule cobaltocene $\left(\mathrm{CoCp}_{2}\right)$, which also acts as a spacer molecule causing almost a doubling of the layer spacings in the $c$-direction upon intercalation. While the intercalation by electronically inert organic guest molecules is reported to reduce the superconducting transition temperature $T_{\mathrm{c}}$ from $7.2 \mathrm{~K}$ in $2 \mathrm{H}-\mathrm{NbSe}_{2}$ to temperatures below $T_{\mathrm{c}}<3 \mathrm{~K}$ [9], an opposite behavior is observed in $\mathrm{NbSe}_{2}\left\{\mathrm{CoCp}_{2}\right\}_{0.26}$ upon intercalation with the electronic donor molecule cobaltocene. $T_{\mathrm{c}}$ is slightly raised with respect to the original host lattice to $T_{\mathrm{c}}^{\text {onset }}=7.5 \mathrm{~K}$. Furthermore, susceptibility, specific heat, and resistivity studies (parallel and perpendicular to the $2 \mathrm{H}-\mathrm{NbSe}_{2}$-layers) of $\mathrm{NbSe}_{2}\left\{\mathrm{CoCp}_{2}\right\}_{0.26}$ reveal unusual pinning properties as well as a non-BCS-like linear $T^{2}$-dependence of $\Delta C / T$ sequences. A re-entrant peak effect, known from the host species $2 \mathrm{H}-\mathrm{NbSe}_{2}$ [10], is also observed. Therefore, $\mathrm{NbSe}_{2}\left\{\mathrm{CoCp}_{2}\right\}_{0.26}$ may be a good candidate for a comparative study to distinguish between different anisotropic superconducting models, like the two-gap model [8] or the anisotropic single-gap model [13].

\section{Sample preparation and characterisation}

$2 \mathrm{H}-\mathrm{NbSe}_{2}$ single crystals have been synthesized via chemical transport in sealed quartz-ampoules (length: $150 \mathrm{~mm}$; inner diameter: $10 \mathrm{~mm}$ ) with iodine as a transport agent in a tubefurnace. The transport reaction was carried out using a temperature gradient: source $\left(T_{1}=840^{\circ} \mathrm{C}\right)$ and sink $\left(T_{2}=\right.$ $\left.600^{\circ} \mathrm{C}\right)$. Plate-like $\mathrm{NbSe}_{2}$ single crystals were isolated and intercalated with cobaltocene $\left(\mathrm{CoCp}_{2}\right)$ in acetonitrile $\left(\mathrm{CH}_{3} \mathrm{CN}\right)$ at a temperature of $65^{\circ} \mathrm{C}$ under inert gas conditions (Ar) for four weeks and characterized by ESEM micrographs, ICPOES (Ionic Coupled Plasma-Optical Emission Spectroscopy), $\mathrm{x}$-ray powder diffraction, and physical property measurements. The sample stoichiometry of one part of the same batch was determined via ICP-OES as $\mathrm{NbSe}_{2}\left\{\mathrm{CoCp}_{2}\right\}_{0.26}$ [14].

Figures $1(a)$ and $(b)$ show an ESEM-micrograph of the crystalline host material $2 \mathrm{H}-\mathrm{NbSe}_{2}$ and of the intercalated $\mathrm{NbSe}_{2}\left\{\mathrm{CoCp}_{2}\right\}_{0.26}$ sample at the same magnification, respectively. Both images show the hexagonal shape of native and intercalated $2 \mathrm{H}-\mathrm{NbSe}_{2}$ crystals. The partial exfoliation of the $\mathrm{NbSe}_{2}$ lattice as a direct consequence of the intercalation process is clearly visible and documents the successful filling of the van der Waals gaps inbetween the individual $\left(\mathrm{NbSe}_{2}\right)$ layers. A subsequent x-ray powder analysis reveals a stageone-type intercalation variant since all the interlayer gaps in $2 \mathrm{H}-\mathrm{NbSe}_{2}$ are at least partially filled.

In figure 2 the powder diffraction pattern of $2 \mathrm{H}-\mathrm{NbSe}_{2}$ is plotted. A strong preferred orientation of the powder samples was observed due to the pronounced two-dimensional shape of the $2 \mathrm{H}-\mathrm{NbSe}_{2}$ crystals. In order to determine precise lattice parameters for the host as well as the intercalated lattice, a Le Bail profile fitting as implemented in the Jana2006 program [15] has been used. The lattice constants of $2 \mathrm{H}$ $\mathrm{NbSe}_{2}$, which crystallizes in the hexagonal space group $P 6_{3} / m m c$, have been determined to $a=0.344570(7) \mathrm{nm}$ and $c=1.25500(4) \mathrm{nm}$. The sharp Bragg-peaks and the absence of additional reflections are in line with the high crystal-quality and purity of the sample. Upon intercalation, the $00 \ell$-series of the host lattice (the blue curve in figure 2) shifts to lower diffraction angles (the green curve in figure 2) in agreement with the expected expansion of the inter-layer spacing, yielding a new $c$-axis parameter of $2.3697(1) \mathrm{nm}$, whereas the $a$-axis expands only slightly to $a=0.346460(7) \mathrm{nm}$. Since each unit 


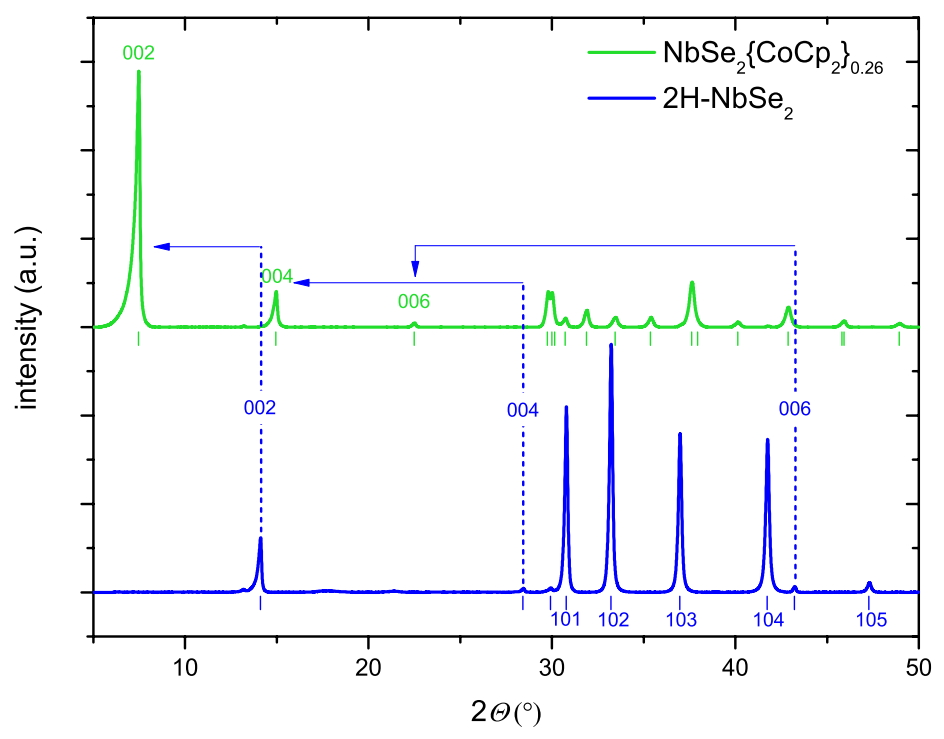

Figure 2. Comparative powder $x$-ray diffraction studies of the $2 \mathrm{H}-\mathrm{NbSe}_{2}$ (blue, bottom) and the intercalated species $\left.2 \mathrm{H}_{-}-\mathrm{NbSe}_{2}\{\mathrm{CoCp}\}_{2}\right\}_{0.26}$ (green, top). The vertical lines below the patterns indicate the refined peak positions according to the space group $P 6_{3} / m m c$ of $2 \mathrm{H}-\mathrm{NbSe}_{2}$ and its intercalated species.

cell contains two $\mathrm{NbSe}_{2}$-layers (with the $\mathrm{Nb}$-atoms located at $z=0.25$ and $z=0.75$, where $z$ is the fractional atomic coordinate along the $c$-axis), the layer expansion corresponds to $\Delta c / 2=0.5574 \mathrm{~nm}$. This is in good agreement with the value published by Dines et al for the layer expansion upon $\mathrm{CoCp}_{2}$ intercalation $(\Delta c=0.556 \mathrm{~nm}[16])$. We note that the Bragg-intensities from the host lattice vanished completely. This suggests that the intercalation process was complete (the green curve in figure 2) in line with an intercalation degree of 0.26 , which is close to the theoretical limit of $x=0.336$ in related dichalcogenides such as $\mathrm{SnSe}_{2}$ [17].

A further indicator for the high quality of the samples is the residual resistivity ratio (RRR). In the case of $2 \mathrm{H}-\mathrm{NbSe}_{2}$ the RRR is determined to be 37 between $7.5 \mathrm{~K}$ and $300 \mathrm{~K}$. This value is higher than most of the values discussed in the literature [18-21]. For the intercalated sample the RRR $=33$ is slightly reduced indicating — if at all-a marginal loss of the crystallinity.

\section{Experimental details}

The diffraction patterns depicted in figure 2 were measured in transmission mode on a HUBER Guinier diffractometer equipped with a Mo- $\mathrm{K}_{\alpha 1}$-source $\left(2 \mathrm{H}-\mathrm{NbSe}_{2}\right)$, or alternatively with a $\mathrm{Cu}-\mathrm{K}_{\alpha 1}$-source $\left(\mathrm{NbSe}_{2}\left\{\mathrm{CoCp}_{2}\right\}_{0.26}\right)$ and a HUBER G670 Guinier camera. The background intensity was removed using the DIFFRACplus EVA software (Bruker AXS, Germany). For better comparison in figure 2 , the $2 \theta_{\text {Mo }}$ axis for the pattern of $2 \mathrm{H}-\mathrm{NbSe}_{2}$ has been rescaled to $\mathrm{Cu}-\mathrm{K}_{\alpha 1}$ radiation.

The DC magnetic susceptibility measurements were performed between $2 \mathrm{~K}$ and $300 \mathrm{~K}$ in an applied magnetic field of up to $5 \mathrm{~T}$ using a commercial SQUID magnetometer (MPMS-7) from Quantum Design. To align the single crystals parallel and perpendicular to the magnetic fields, small single crystalline plates ( $\leqslant 4 \mathrm{~mm} \times 2.5 \mathrm{~mm}$ edge lengths) were embedded in $\mathrm{NaCl}$ powder ( $5 \mathrm{~N}$; Alfa Aesar) and confined by two quartz glass rods inside a straw to prevent sample reorientation during the measurement. The accuracy of the individual crystal orientation with respect to the field is estimated to be better than $\pm 5^{\circ}$. For the AC susceptibility we used a SQUID Magnetometer (MPMS-5) with an ACoption, and for magnetic fields up to $14 \mathrm{~T}$ a state of the art SQUID-VSM system from Quantum Design was utilized. The specific heat experiments were performed with a commercial physical property measurement system (PPMS) equipment between $2 \mathrm{~K}$ and $300 \mathrm{~K}$ in magnetic fields up to $5 \mathrm{~T}$. For the electrical transport measurements we performed a four probe method using the PPMS. To avoid Lorentz currents, the external magnetic field and the current flow were applied in the same direction parallel to the layers. The dimensions of the single crystal employed for the resistivity measurements were $3.4 \mathrm{~mm} \times 0.6 \mathrm{~mm}$ (thickness $d \approx 150 \mu \mathrm{m}$ ), and the distance between the current and voltage contacts (size $\approx$ $0.6 \mathrm{~mm} \times 0.4 \mathrm{~mm}$ ) was $\approx 2.4 \mathrm{~mm}$ and $0.7 \mathrm{~mm}$, respectively.

\section{Experimental results and discussion}

\subsection{Susceptibility}

The temperature dependent magnetic dc-susceptibility $\left(\chi_{V}(T)\right)$ of $\mathrm{NbSe}_{2}\left\{\mathrm{CoCp}_{2}\right\}_{0.26}$ is depicted in figure 3 for zero field cooled (ZFC) and field cooled (FC) cycles in a low magnetic field applied perpendicular and parallel to the $a b$-planes. For the ZFC sequence the sample was first cooled down to $2 \mathrm{~K}$ in a zero field, where the earth magnetic field was compensated within an error bar of $1 \mu \mathrm{T}$ by the so-called TinyBee setup described in detail in [22]. In the subsequent heating run a small magnetic field of $\mu_{0} H=0.5 \mathrm{mT}$ was applied to record the susceptibility data up to $8 \mathrm{~K}$. For the susceptibility measurements with $H$ perpendicular to the $a b$-plane $(H \perp a b)$ a demagnetization factor of $N=0.8$ was taken into account. This factor is estimated experimentally by measuring a lead 


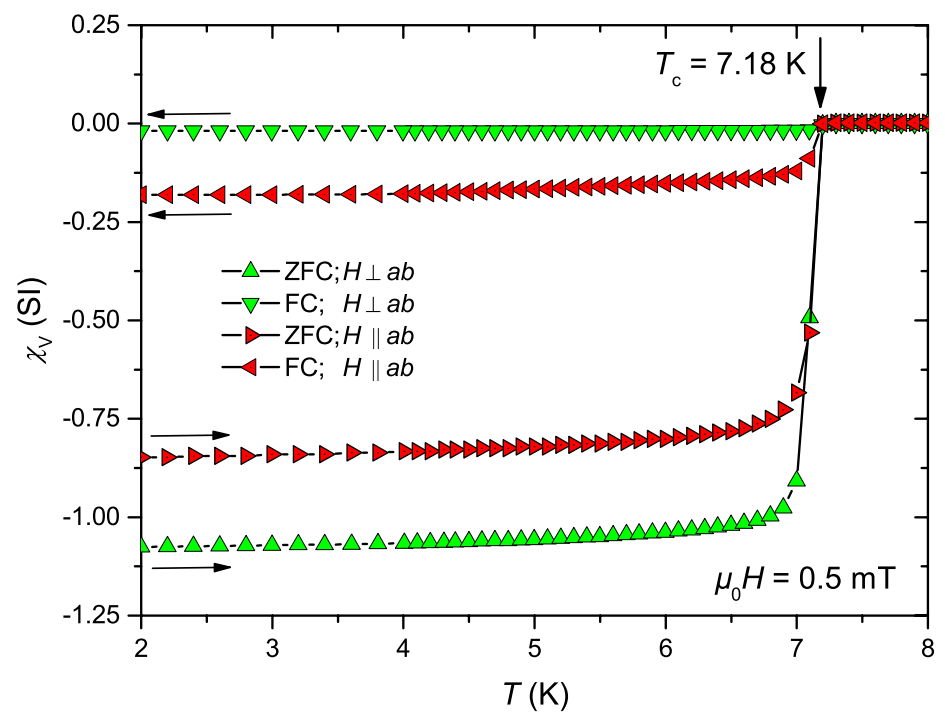

Figure 3. ZFC and FC volume susceptibility $\left(\chi_{V}\right)$ of $\mathrm{NbSe}_{2}\left\{\mathrm{CoCp}_{2}\right\}_{0.26}$ with $\mu_{0} H$ perpendicular and parallel to the $a b$-planes. For the measurement with $H \perp a b$ a demagnetization factor of $N=0.8$ was considered.

plate with a shape nearly identical to the investigated sample $(\approx 4 \mathrm{~mm} \times 2.5 \mathrm{~mm}$; slight trapezoid). For the volume susceptibility $\chi_{V}$ we calculated the density of $\mathrm{NbSe}_{2}\left\{\mathrm{CoCp}_{2}\right\}_{0.26}$ from the lattice parameters and the stoichiometry of the sample known from the ICP-OES and elementary analysis, resulting in a value of $\rho=4.052 \mathrm{~g} \mathrm{~cm}^{-3}$.

In figure 3 all $\chi_{V}(T)$ curves exhibit a sharp superconducting transition at $T_{\mathrm{c}}=7.18 \mathrm{~K}$ for the $\mathrm{ZFC}$ and FC procedures. This critical temperature is virtually identical to the one of the host substance and is slightly smaller than $T_{\mathrm{c}}^{\text {onset }}=7.5 \mathrm{~K}$ estimated from our resistivity studies (section 4.3, figure $7(a)$ ). This result is remarkable due to the fact that previous intercalations of $2 \mathrm{H}-\mathrm{NbSe}_{2}$ with electronically innocent guest molecules yielded a significant reduction of $T_{\mathrm{c}}$ and values between $0.6 \mathrm{~K}$ and $6.88 \mathrm{~K}[9,19,21,23,24]$.

Furthermore, the $\chi_{V}(T)$-data of $\mathrm{NbSe}_{2}\left\{\mathrm{CoCp}_{2}\right\}_{0.26}$ show some deviation from an ideal bulk superconducting behavior. In the ideal case, the ZFC procedure in low magnetic fields results in a complete shielding state of the sample with $\chi_{V}=-1$ at low temperatures. The FC measurement provides information about the strength of the flux expulsion due to the Meissner effect. In the case of hard type-II superconductors, such as the high temperature superconductor $\mathrm{YBaCuO}$ [25], a superconducting volume susceptibility in the range of $-1 \leqslant$ $\chi_{V} \leqslant-0.5$ is usually expected, in contrast to our findings for $\mathrm{NbSe}_{2}\left\{\mathrm{CoCp}_{2}\right\}_{0.26}$ with $\chi_{V} \approx-0.05(H \perp a b)$.

For $\mathrm{NbSe}_{2}\left\{\mathrm{CoCp}_{2}\right\}_{0.26}$ we observed in the case of $H \| a b$ that the ZFC procedure results in a partial screening $\left(\chi_{V}>\right.$ $-1)$, mainly due to the presence of a normal state region in the van der Waals gap between the $\left(\mathrm{NbSe}_{2}\right)_{2}$ layers. On the other hand for $H \perp a b$ a full screening is expected. The deviation of $7 \%$ from the ideal value may be due to the error in the determination of the demagnetization factor or the sample density. For the FC measurement with $H \| a b$ the sample is exposed to a maximal field of $\mu_{0} H=0.5 \mathrm{mT}$ due to the vanishing demagnetization factor. This leads to a flux expulsion in the superconducting planes but not in the inter-layer space. Therefore, the resulting intermediate phase gives rise to a small but not negligible Meissner-phase. On the contrary, for $H \perp a b$ the demagnetization factor is close to one, due to the plate-like shape of the sample. This may lead to local magnetic fields higher than $\mu_{0} H_{\mathrm{c} 1 \perp}$, resulting in fluxpenetration with vortex field strengths higher than $\mu_{0} H_{\mathrm{c} 1 \perp}$. As a result, the Meissner effect may be superimposed by this flux pinning process and therefore only a negligible Meissner effect is observed.

This deviation from an ideal superconducting behavior strongly points to a pronounced anisotropic superconductor with a layered structure of superconducting planes which are weakly coupled by van der Waals forces.

\subsection{Critical magnetic fields and Ginzburg-Landau parameters}

In order to calculate the microscopic superconducting parameters like the London penetration depth $(\lambda)$, the GL coherence length $(\xi)$, and the GL parameter $(\kappa)$, the lower and upper critical fields perpendicular and parallel to the $a b$-planes are determined from magnetization curves and from temperature dependent specific heat and resistivity measurements in various magnetic fields.

The lower critical fields of $2 \mathrm{H}-\mathrm{NbSe}_{2}$ and $\mathrm{NbSe}_{2}$ $\left\{\mathrm{CoCp}_{2}\right\}_{0.26}$ are estimated from magnetization measurements $\mu\left(\mu_{0} H\right)$ at very low magnetic fields, using the high resolution of $\mu_{0} \Delta H=1 \mu \mathrm{T}$ increments accomplished by the TinyBee setup [22]. In figure $4(a)$ the magnetic moment versus magnetic field for $2 \mathrm{H}-\mathrm{NbSe}_{2}$ in the case of $H$ perpendicular to the $a b$-planes at $2 \mathrm{~K}$ is plotted in the range between 0 and $4 \mathrm{mT}$ using minute increments of $\mu_{0} \Delta H=0.025 \mathrm{mT}$. The linear fit (employing $\mu(H)$ data below $0.5 \mathrm{mT}$ ) shows the first deviation from the magnetization curve at approximately $1 \mathrm{mT}$. According to a common procedure in the literature (see, e.g. [26]) the latter value can therefore be employed to specify $\mu_{0} H_{\mathrm{c} 1}$ at $2 \mathrm{~K}$. This is in accordance with the kink in the 

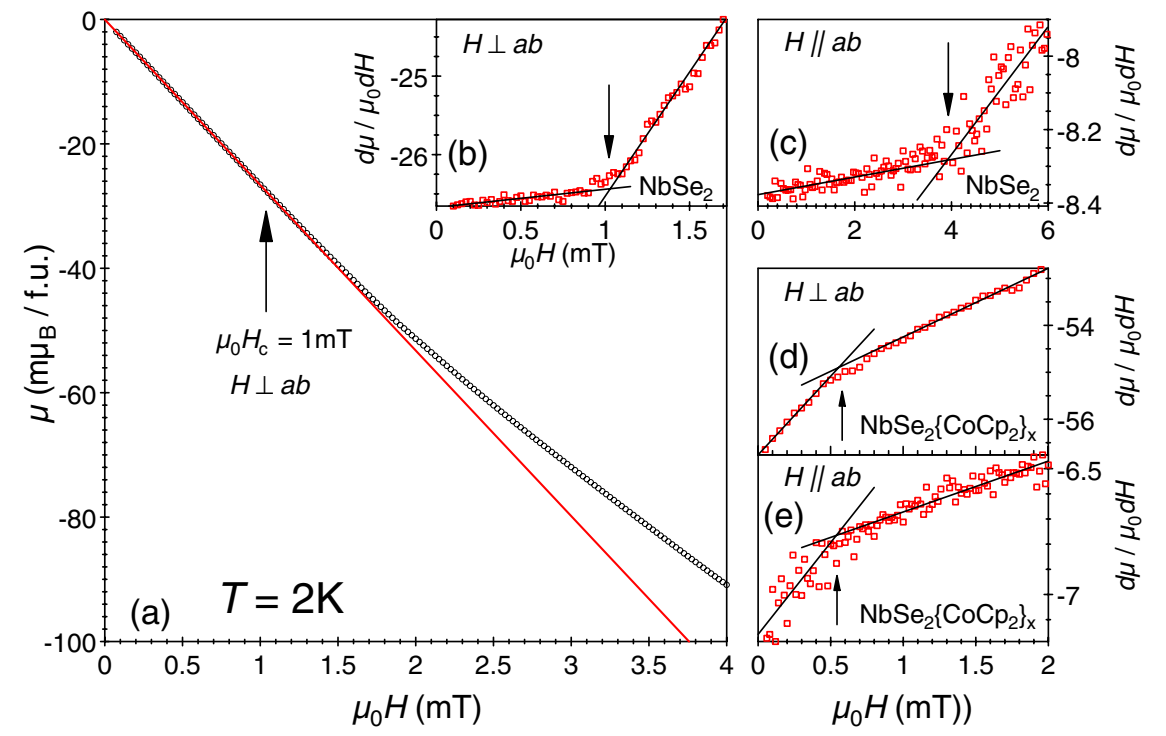

Figure 4. (a) The initial magnetization curve $\mu\left(\mu_{0} H\right)$ up to $4 \mathrm{mT}$ of $\mathrm{NbSe}_{2}$ with $H$ perpendicular to the $a b$-planes at $T=2 \mathrm{~K}$. The red line is a linear fit to the magnetization data below $0.5 \mathrm{mT}$. In the insert $(b)$ the first derivative of the magnetic moment $\mu$ with respect to the magnetic field $\mu_{0} H$ is plotted versus $\mu_{0} H$ in the range between 0 and $1.7 \mathrm{mT}$. The solid lines are linear fits to the data. The point of intersection defines $\mu_{0} H_{\mathrm{c}}$. Such a plot is also shown in $(c)$ for $\mathrm{NbSe}_{2}$ with $H$ parallel to the $a b$-planes and in $(d)$ and $(e)$ for $\mathrm{NbSe}_{2}\left\{\mathrm{CoCp}_{2}\right\}_{x}$ $(x=0.26)$ with $H$ perpendicular and parallel to the $a b$-planes, respectively.

derivative $\frac{\mathrm{d} \mu}{\mu_{0} \mathrm{~d} H}$, as shown in figure $4(b)$. This kink in the derivative $\frac{\mathrm{d} \mu}{\mu_{0} \mathrm{~d} H}$ is, however, a more stringent criterion for the determination of $\mu_{0} H_{\mathrm{c} 1}$, because its location is less dependent on the choice and size of the fitting range and was therefore used to extract the $H_{\mathrm{c} 1}$ values. This assessment is a sensitive indicator to identify the magnetic field $\mu_{0} H_{\mathrm{c} 1}$, which is defined as the field where the first flux in the form of a quantized vortex penetrates the sample and starts to increase continuously with an increasing magnetic field [1]. It should be mentioned that for $\mathrm{NbSe}_{2}, \frac{\mathrm{d} \mu}{\mu_{0} \mathrm{~d} H}\left(\mu_{0} H\right)$ shows a slight increase for small fields in both field orientations, which may be due to a small flux penetration caused by sample inhomogeneities, thereupon the magnetic flux penetrates the sample in the form of quantized vortexes visible by a pronounced increase in the derivative $\frac{\mathrm{d} \mu}{\mu_{0} \mathrm{~d} H}$, as shown in figures $4(b)$ and $(c)$.

In contrast to this, figures $4(d)$ and $(e)$ show for the intercalated sample $\mathrm{NbSe}_{2}\left\{\mathrm{CoCp}_{2}\right\}_{0.26}$ a strong initial flux penetration. At a distinct magnetic field the slope of the derivative $\frac{\mathrm{d} \mu}{\mu_{0} \mathrm{~d} H}$ changes to smaller values indicating a reduced rate of flux penetration. This unusual behavior hints at unquantized flux penetration in the intercalated $2 \mathrm{H}-\mathrm{NbSe}_{2}$ already below $H_{\mathrm{c} 1}$. This observation is likely explained by the large spacial separation of the superconducting layers. In the case of magnetic fields above $H_{\mathrm{c} 1}$, the screening currents become sufficiently large to channel the flux into quantized vortices. Since the spacial extent of a vortex, given by the penetration depth $\lambda$, is far larger than the layer distance in $2 \mathrm{H}-\mathrm{NbSe}_{2}$, the vortex penetration rate above $H_{\mathrm{c} 1}$ remains essentially unaffected by the subtle and additional increase in the inter-layer distance due to the intercalation of the cobaltocene molecules.

The lower critical field $\mu_{0} H_{\mathrm{c} 1}$ at zero temperature is determined by extrapolating the magnetic field data at $2 \mathrm{~K}$ and
$T_{\mathrm{c}}$, using the empiric parabolic relation [27]

$$
\mu_{0} H_{\mathrm{c} 1}(T)=\mu_{0} H_{\mathrm{c} 1}(0) \times\left[1-\left(T / T_{\mathrm{c}}\right)^{2}\right]
$$

by taking into account a demagnetization factor of $N=$ 0.8 for the applied field $H$ perpendicular to the $a b$ planes. The resulting lower critical field values with $H$ parallel and perpendicular to the ab-planes are depicted in figures $4(b)-(e)$ and are summarized in table 1 for $\mathrm{NbSe}_{2}$ and $\mathrm{NbSe}_{2}\left\{\mathrm{CoCp}_{2}\right\}_{0.26}$, respectively.

The upper critical fields $H_{\mathrm{c} 2}$ perpendicular and parallel to the $a b$-planes are extracted from specific heat and resistivity measurements, respectively (for details see below). For $\mathrm{NbSe}_{2}\left\{\mathrm{CoCp}_{2}\right\}_{0.26}$, the electronic contribution to the specific heat divided by temperature $\Delta C / T$ is plotted for $H$ perpendicular to the $a b$-planes versus $T^{2}$ in various magnetic fields up to $5 \mathrm{~T}$ in figure $5(a)$. The critical temperature is suppressed gradually by the magnetic field from $T_{\mathrm{c}}=7.12 \mathrm{~K}$ to a temperature below $2 \mathrm{~K}$ for $\mu_{0} H>5 \mathrm{~T}$. The specific heat discontinuity at $T_{\mathrm{c}}(0)$ of $\delta C / T \simeq 31.6 \mathrm{~mJ} \mathrm{~mol}^{-1} \mathrm{~K}^{2}$ divided by the Sommerfeld coefficient in the normal state $\gamma \simeq$ $22.1 \mathrm{~mJ} \mathrm{~mol}^{-1} \mathrm{~K}^{2}$ yields the thermodynamic ratio $\delta C / \gamma T_{\mathrm{c}}=$ 1.43 , which surprisingly matches the expected value of 1.43 for a weakly coupled BCS superconductor [28]. In contrast to this, a somewhat higher $\delta C / \gamma T_{\mathrm{c}}$ ratio is observed in the parent compound $2 \mathrm{H}-\mathrm{NbSe}_{2}$ with varying values between 1.96 and 2.12 , which may be connected to a more pronounced electronphonon coupling (see table 1 and $[29,30]$ ). Such enhanced values are generally known for layered superconductors, e.g. FeSe $\left(\delta C / \gamma T_{\mathrm{c}}=1.65\right)[31]$.

Furthermore, it should be mentioned that the nearly linear trend in the $\triangle C / T$ versus $T^{2}$ plot below $T_{\mathrm{c}}$ of $\mathrm{NbSe}_{2}\left\{\mathrm{CoCp}_{2}\right\}_{0.26}$ (figure 5(a)) is also observed in the parent species $[13,29,30]$. This linearity is unusual for single band $s$-wave superconductors. A similar superconducting behavior 
Table 1. Overview of the superconducting parameters of $2 \mathrm{H}-\mathrm{NbSe}_{2}$ and the intercalated species $\mathrm{NbSe}_{2}\left\{\mathrm{CoCp}_{2}\right\}_{0.26}$ from this work, in comparison with selected studies from the literature [8, 40-42].

\begin{tabular}{|c|c|c|c|c|c|c|}
\hline & $\begin{array}{l}2 \mathrm{H}-\mathrm{NbSe}_{2} \\
{[40]}\end{array}$ & $\begin{array}{l}2 \mathrm{H}-\mathrm{NbSe}_{2} \\
{[41]}\end{array}$ & $\begin{array}{l}2 \mathrm{H}-\mathrm{NbSe}_{2} \\
{[42]}\end{array}$ & $\begin{array}{l}2 \mathrm{H}-\mathrm{NbSe}_{2} \\
{[8]}\end{array}$ & $\begin{array}{l}2 \mathrm{H}-\mathrm{NbSe}_{2} \\
\text { this work }\end{array}$ & $\begin{array}{l}\mathrm{NbSe}_{2}\left\{\mathrm{CoCp}_{2}\right\}_{0.26} \\
\text { this work }\end{array}$ \\
\hline$\overline{T_{\mathrm{c}}}$ & $7.38 \mathrm{~K}$ & $7.07 \mathrm{~K}$ & $7.15 \mathrm{~K}$ & $7.15 \mathrm{~K}$ & $7.15 \mathrm{~K}$ & $7.35 \mathrm{~K}$ \\
\hline $\begin{array}{l}\mu_{0} H_{\mathrm{c} 2 \|} \\
\mu_{0} H_{\mathrm{c} 2 \perp} \\
\mu_{0} H_{\mathrm{c} 1 \|} \\
\mu_{0} H_{\mathrm{c} 1 \perp}\end{array}$ & $4.3 \mathrm{~T}^{\star}$ & $\begin{array}{l}12.5 \mathrm{~T} \\
3.65 \mathrm{~T}\end{array}$ & $\begin{array}{l}17.3 \mathrm{~T} \\
5.3 \mathrm{~T}\end{array}$ & $\begin{array}{l}12.3 \mathrm{~T} \\
4.1 \mathrm{~T} \\
3.9 \mathrm{mT} \\
9 \mathrm{mT}\end{array}$ & $\begin{array}{l}14.1 \mathrm{~T} \\
4.3 \mathrm{~T} \\
4.24 \mathrm{mT} \\
5.49 \mathrm{mT}\end{array}$ & $\begin{array}{l}18.5 \mathrm{~T} \\
4.2 \mathrm{~T} \\
0.58 \mathrm{mT} \\
3.13 \mathrm{mT}\end{array}$ \\
\hline $\begin{array}{l}\lambda_{\|} \\
\lambda_{\perp}\end{array}$ & $\begin{array}{l}69 \mathrm{~nm} \\
230 \mathrm{~nm}\end{array}$ & & & $\begin{array}{l}265 \mathrm{~nm} \\
797 \mathrm{~nm}\end{array}$ & $\begin{array}{l}330 \mathrm{~nm} \\
524 \mathrm{~nm}\end{array}$ & $\begin{array}{l}455 \mathrm{~nm} \\
3561 \mathrm{~nm}\end{array}$ \\
\hline $\begin{array}{l}\xi_{\|} \\
\xi_{\perp}\end{array}$ & $\begin{array}{l}7.7 \mathrm{~nm} \\
2.3 \mathrm{~nm}\end{array}$ & $\begin{array}{l}9.5 \mathrm{~nm} \\
2.8 \mathrm{~nm}\end{array}$ & $\begin{array}{l}7.88 \mathrm{~nm} \\
2.42 \mathrm{~nm}\end{array}$ & $\begin{array}{l}9 \mathrm{~nm} \\
3 \mathrm{~nm}\end{array}$ & $\begin{array}{l}8.75 \mathrm{~nm} \\
2.67 \mathrm{~nm}\end{array}$ & $\begin{array}{l}8.86 \mathrm{~nm} \\
2.02 \mathrm{~nm}\end{array}$ \\
\hline $\begin{array}{l}\kappa_{\|} \\
\kappa_{\perp}\end{array}$ & $\begin{array}{l}30 \\
9\end{array}$ & $\begin{array}{l}72 \\
21\end{array}$ & $\begin{array}{l}29 \\
11\end{array}$ & $\begin{array}{l}86 \\
30\end{array}$ & $\begin{array}{l}86 \\
38\end{array}$ & $\begin{array}{l}300 \\
51\end{array}$ \\
\hline$\delta C / \gamma T_{\mathrm{c}}$ & 1.97 [13] & 1.96 & 2.14 & 2.12 [29] & 2.05 & 1.43 \\
\hline
\end{tabular}

Note: The symbols $\|$ and $\perp$ refer to directions parallel and perpendicular to the $a b$ plane, respectively. The * marks the upper critical fields $B_{\mathrm{c} 2 \perp}$, which is estimated from figure 2 in [40].
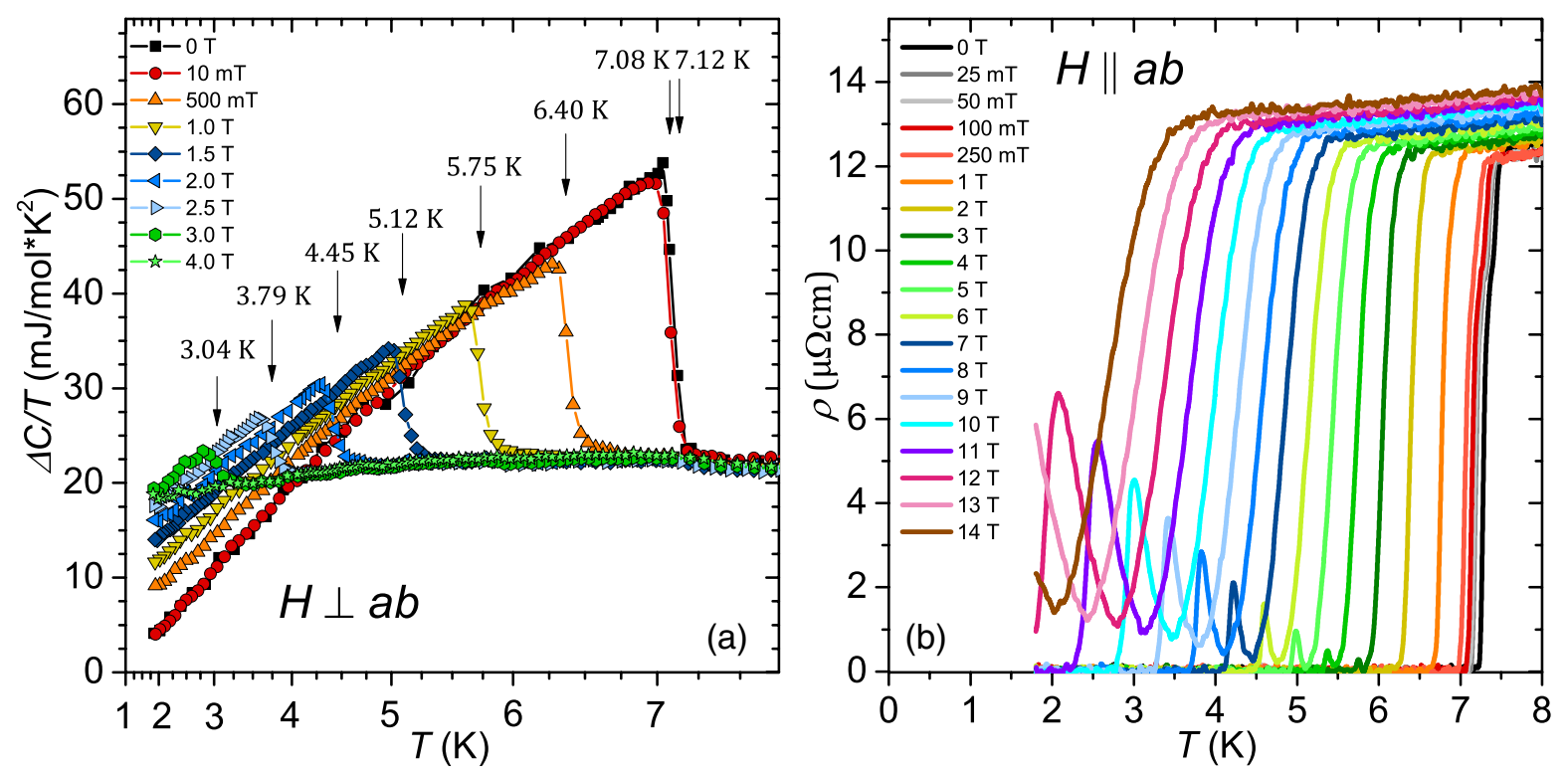

Figure 5. (a) Temperature-dependent electronic contribution of the specific heat plotted as $\Delta C / T$ versus $T^{2}$ of $\mathrm{NbSe}_{2}\left\{\mathrm{CoCp}_{2}\right\}_{0.26}$ for various magnetic fields (as labeled) perpendicular to the $a b$-planes. (b) Temperature-dependent electrical resistivity for various magnetic fields (as labeled) parallel to the $a b$-planes.

is also found in the intermetallic compound $\mathrm{MgB}_{2}$, which is discussed with respect to a second superconducting band at the Fermi level [32]. In particular, for the host sample $2 \mathrm{H}-\mathrm{NbSe}_{2}$, Huang et al proposed that a two-gap scenario is more favorable than an anisotropic $s$-wave model [29]. We will outline in the following that the latter proposal is not supported by our own study. We give a more detailed discussion and comparison to the model calculations in section 5 .

For $\mathrm{NbSe}_{2}\left\{\mathrm{CoCp}_{2}\right\}_{0.26}$ the temperature dependency of the in-plane resistivity in various magnetic fields is shown in figure $5(b)$ in the temperature range between $1.8 \mathrm{~K}$ and $8 \mathrm{~K}$. Towards lower temperatures, after the initial steep drop of the resistivity due to the onset of superconductivity, the sample shows a finite resistivity, which again vanishes on further cooling. This observed reentrant behavior is also known from the host sample $2 \mathrm{H}-\mathrm{NbSe}_{2}$ [33]. The out-of-plane resistivity behavior could, however, not be determined, although a special set-up was used as described in [34].

In figure 6 the phase diagrams of the upper $(a)$ and lower (b) critical magnetic fields parallel and perpendicular to the $a b$-planes are presented. For $\mu_{0} H_{\mathrm{c} 2 \|}$ the critical temperatures are obtained from the $50 \%$ values of the normal state resistivity (figure $5(b)$ ) slightly below the onset of superconductivity. For $H_{\mathrm{c} 2 \perp}$ the $T_{\mathrm{c}}$ data are determined at that temperature, where the specific heat discontinuity of the superconducting anomaly reaches half of the values $\delta C / T$ (figure 5(a)). For both branches $(H \| a b$ and $H \perp a b$ in figure 6(a)) we extrapolate the $\mu_{0} H_{\mathrm{c} 2}$ values in the linear region between 2 and $6 \mathrm{~K}$ down to zero temperature taking into account the WHH-theory [35]. It should be mentioned that a positive curvature of $\mu_{0} H_{\mathrm{c} 2 \|}(T)$ is observed just below $T_{\mathrm{c}}$, which is not in line with the WHHtheory. However, this feature appears to be a characteristic 

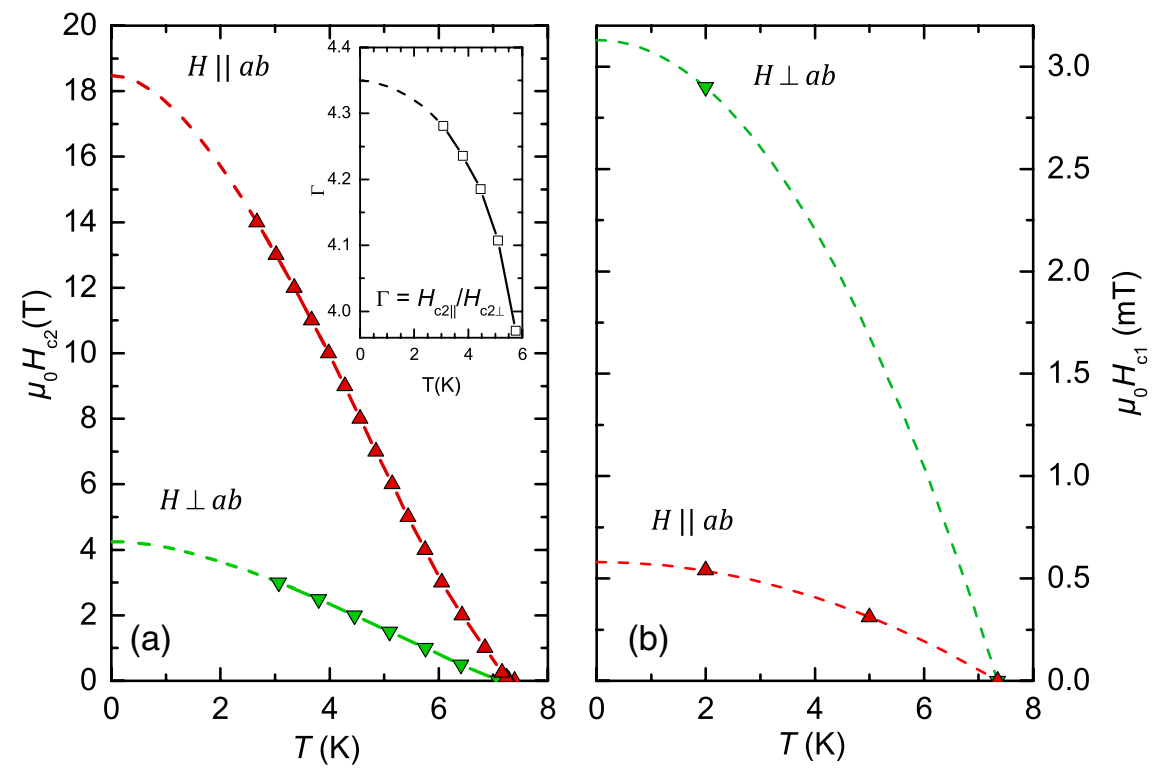

Figure 6. (a) Temperature dependence of the upper critical magnetic field, $\mu_{0} H_{\mathrm{c} 2}$, and $(b)$ of the lower critical field, $\mu_{0} H_{\mathrm{c} 1}$, perpendicular and parallel to the $\mathrm{NbSe}_{2}$ planes of $\mathrm{NbSe}_{2}\left\{\mathrm{CoCp}_{2}\right\}_{0.26}$. The insert displays the temperature dependent anisotropy factor $\Gamma$. The solid lines are guides for the eyes. The dashed lines are fits to the data according to the WHH-theory [35] for $\mu_{0} H_{\mathrm{c} 2}(T)$ and to the empiric relation (see equation (1)) for $\mu_{0} H_{\mathrm{c} 1}(T)$, respectively.

$H_{\mathrm{c} 2 \|}(T)$ signature observed in many layered superconductors (see chapter 6 in [1]), and in particular also in the host substance $2 \mathrm{H}-\mathrm{NbSe}_{2}$ [8]. This positive, upward curvature may be due to the onset of a dimensional cross-over [36], or caused by an anisotropic Fermi-surface [20]. The phase boundaries of the lower critical fields down to zero temperatures are generated from the values of the lower critical fields at $2 \mathrm{~K}$ (see figures $4(d)$ and $(e), 5 \mathrm{~K}$ and from the $T_{\mathrm{c}}$ values at $H=0$, using the empiric relation for $\mu_{0} H_{\mathrm{c} 1}(T)$ (see equation (1)). The resulting lower and upper critical field values at $T=0 \mathrm{~K}$ are tabulated in table 1, in comparison with selected results of the host sample $2 \mathrm{H}-\mathrm{NbSe}_{2}$.

The upper critical field ratio $H_{\mathrm{c} 2 \|} / H_{\mathrm{c} 2 \perp}$ of $\mathrm{NbSe}_{2}$ $\left\{\mathrm{CoCp}_{2}\right\}_{0.26}$ defines the anisotropy factor $\Gamma$, which is about 4.4 at $T=0$. This value is $34 \%$ larger than that of $2 \mathrm{H}-\mathrm{NbSe}_{2}$ and is similar to those values found in different single crystalline $\mathrm{MgB}_{2}$ samples (for an overview see [37]). For both species, $\mathrm{NbSe}_{2}$ and $\mathrm{MgB}_{2}$, a multi-band scenario is discussed in the literature, which goes along with a temperature dependent anisotropy factor $\Gamma(T)[8,37]$. Such behavior is also observed in $\mathrm{NbSe}_{2}\left\{\mathrm{CoCp}_{2}\right\}_{0.26}$ (insert of figure 6(a)), but the change of the anisotropy per Kelvin $\frac{\mathrm{d} \Delta \Gamma}{\mathrm{d} T}$ of approximately $0.075 \mathrm{~K}^{(-1)}$ is two times smaller than in $\mathrm{MgB}_{2}$ - the benchmark system for multi-band superconduction. This may be due to different determination methods of $\Gamma(T) \quad[8,37,38]$. Even though the change of $\Gamma(T)$ is smaller in comparison to $\mathrm{MgB}_{2}$, this criterion does not disqualify $\mathrm{NbSe}_{2}\left\{\mathrm{CoCp}_{2}\right\}_{0.26}$ as suitable candidate for a multi-band scenario at this stage of our analysis.

For a detailed comparison between $2 \mathrm{H}-\mathrm{NbSe}_{2}$ and $\mathrm{NbSe}_{2}\left\{\mathrm{CoCp}_{2}\right\}_{0.26}$ we also calculated the microscopic superconducting parameters $\xi, \lambda$, and $\kappa$ directly from the critical magnetic fields according to the anisotropic GL theory [1]. Here the GL coherence length $\xi_{\mathrm{GL}}$ is extracted from the upper critical field using the following equations:

$\mu_{0} H_{\mathrm{c} 2 \|}=\frac{\Phi_{0}}{2 \pi \xi_{\|} \xi_{\perp}} \quad$ and $\quad \mu_{0} H_{\mathrm{c} 2 \perp}=\frac{\Phi_{0}}{2 \pi \xi_{\|}^{2}}$

with the flux quantum $\Phi_{0}=h / 2 e$. The London penetration depth $\lambda$ is extracted from the lower critical field:

$\mu_{0} H_{\mathrm{c} 1 \|}=\frac{\Phi_{0}}{4 \pi \lambda_{\|} \lambda_{\perp}} \times \ln \kappa_{\|} \quad$ and $\quad \mu_{0} H_{\mathrm{c} 1 \perp}=\frac{\Phi_{0}}{4 \pi \lambda_{\|}^{2}}$
$\times \ln \kappa_{\perp}$

with the GL-parameters

$$
\kappa_{\|}=\left(\frac{\lambda_{\|} \lambda_{\perp}}{\xi_{\|} \xi_{\perp}}\right)^{1 / 2} \quad \text { and } \quad \kappa_{\perp}=\frac{\lambda_{\|}}{\xi_{\|}}
$$

which are extracted from microscopic superconducting parameters.

All the quantities are presented in table 1 together with those of selected studies in order to compare our results of the host species $2 \mathrm{H}-\mathrm{NbSe}_{2}$ with former findings in the literature (see, [8, 40-42]). First of all it should be mentioned that within the variation of different calculations and measurement procedures, the superconducting quantities like $\xi, \lambda$, and $\kappa$ of the parent compound $2 \mathrm{H}-\mathrm{NbSe}_{2}$ are in good agreement with previous results. The direct comparison between the host species and $\mathrm{NbSe}_{2}\left\{\mathrm{CoCp}_{2}\right\}_{0.26}$ surprisingly reveals that the microscopic superconducting GL quantities, $\xi_{\|}$and $\lambda_{\|}$, remain almost unchanged within the layers upon $\mathrm{CoCp}_{2}$ intercalation. On the contrary, the coherence length perpendicular to the layers $\xi_{\perp}$ diminishes by $25 \%$ after $\mathrm{CoCp}_{2}$ intercalation, and is of the order of the inter-layer distance of the intercalated species. In addition, the penetration depth parallel to the layers $\lambda_{\|}$is approximately of the same order of magnitude for both species, whereas for $\lambda_{\perp}$ a drastic increase by a factor of 
4.5 is observed between the host and the intercalated sample, respectively.

This analysis emphasizes the outstanding character of the intercalated samples, as the superconducting properties within the layers remain almost unchanged but change significantly perpendicular to the layer along the $c$ lattice parameter. This is a surprising result since cobaltocene $\left(\mathrm{CoCp}_{2}\right)$ is an electronic donor molecule, which is capable of transferring its unpaired electron into the conduction band of suitable host materials which might act as electron acceptors. Indeed, the electron transfer is indicated by an enhancement of the Sommerfeld coefficient $\gamma$ in the intercalated sample $\left(\mathrm{NbSe}_{2}: \gamma=18 \mathrm{~mJ} \mathrm{~mol}^{-1} \cdot \mathrm{K}^{-2} ; \mathrm{NbSe}_{2}\left\{\mathrm{CoCp}_{2}\right\}_{0.26}: \gamma=\right.$ $22 \mathrm{~mJ} \mathrm{~mol}^{-1} \cdot \mathrm{K}^{-2}$ ) and a vanishing local magnetic moment at the intercalated cobaltocene molecules in the normal state. However, the superconducting properties of the parent lattice do not change significantly within the layers upon the intercalation.

Within the framework of the anisotropic GL theory, it is expected for a single gap anisotropic superconductor that the ratio of the upper critical field $H_{\mathrm{c} 2 \|} / H_{\mathrm{c} 2 \perp}$ equals the ratio of $\xi_{\|} / \xi_{\perp}, \lambda_{\perp} / \lambda_{\|}$and $\kappa_{\|} / \kappa_{\perp}$ [1]. The anisotropy factor $\Gamma \approx 3.3$ of $2 \mathrm{H}-\mathrm{NbSe}_{2}$ can be extracted from its $H_{\mathrm{c} 2}$ - and $\xi$-ratio. In $2 \mathrm{H}-\mathrm{NbSe}_{2}$, the $\xi$-ratio is slightly larger than the corresponding $\lambda$ - (1.6) and $\kappa$-ratio (2.2). For the intercalated sample $\mathrm{NbSe}_{2}\left\{\mathrm{CoCp}_{2}\right\}_{0.26}$ we observe a higher anisotropy factor of about 4.4 , which is equal to the $\xi$ ratio, but is significantly smaller than the $\lambda$ - (7.8) and $\kappa$-ratio (5.9). These results therefore suggest that the anisotropic superconducting behavior in $\mathrm{NbSe}_{2}\left\{\mathrm{CoCp}_{2}\right\}_{0.26}$ cannot be described and modeled within the framework of the anisotropic GL theory involving an anisotropy in the effective masses [1]. This is especially true for a pronounced anisotropic system where the layer distance is of the same order of magnitude as the coherence length [10]. An alternative approach for the description of a layered superconductor based on Josephsoncoupled 2D layers leads to a somewhat different expression for $H_{\mathrm{c} 1 \|}$ [39]. However, the numerical values for the $\lambda$ - and $\kappa$-ratio for $\mathrm{NbSe}_{2}\left\{\mathrm{CoCp}_{2}\right\}_{0.26}$ are almost identical to those obtained above.

\subsection{Peak effect}

As mentioned in section 4.2 the temperature-dependent inplane resistivity in various magnetic fields shows a reentrant superconducting behavior (see figure $5(b)$ ). This behavior is directly associated with a peak effect which is frequently found in layered type-II superconductors, including the host sample $2 \mathrm{H}-\mathrm{NbSe}_{2}$ (see, for example, [43]). The observed peaks in the resistivity data $\rho(T)$ in figure $5(b)$ might be a consequence of two competing mechanisms: superconductivity and flux motion controlled resistivity. Therefore, an analysis of the temperature-dependent resistivity could provide a measure of the average vortex velocity. For conventional low temperature superconductors this effect is primarily observed in the regime of the upper critical field $\mu_{0} H_{\mathrm{c} 2}$ and indicates the presence of different superconducting phases arising from a change of flux motion [44]. For example, a highly dense vortex liquid phase is expected below $\mu_{0} H_{\mathrm{c} 2}$, when the layer separation, $d$, is of the same order of magnitude as the coherence length $\xi$ [10]. For $\mathrm{NbSe}_{2}\left\{\mathrm{CoCp}_{2}\right\}_{0.26}$ this criterion is nearly fulfilled when the magnetic field is applied perpendicular to the layers. In that case $d$ becomes compatible in magnitude with $\xi_{\perp} / 2$ (see table 1).

In order to deal more precisely with the temperature dependent resistivity in the superconducting phase of $\mathrm{NbSe}_{2}\left\{\mathrm{CoCp}_{2}\right\}_{0.26}$ we present in figures 7(a) and $(b)$ enlarged plots of $\rho(T)$ near $T_{\mathrm{c}}$ in two selected magnetic fields, $0 \mathrm{~T}$ and $10 \mathrm{~T}$, respectively. In a zero magnetic field, $\mathrm{NbSe}_{2}\left\{\mathrm{CoCp}_{2}\right\}_{0.26}$ exhibits the highest superconducting critical temperature, $T_{\mathrm{c}}^{\text {onset }}=7.5 \mathrm{~K}$, observed so far in the intercalated or substituted $2 \mathrm{H}-\mathrm{NbSe}_{2}$ samples (see section 4.1). Towards lower temperatures and after the initial superconducting drop, the resistivity shows a pronounced shoulder around $7.4 \mathrm{~K}$, followed again by a steep drop until $\rho(T)$ vanishes on further cooling. This observation hints at two competing mechanisms which control the $\rho(T)$ behavior of the intercalated $\mathrm{NbSe}_{2}$ phases. In order to identify this additional contribution a single superconducting transition has been modeled by a Boltzmann-type sigmoidal function to fit the experimental data (disregarding the temperature region between $7.2 \mathrm{~K}$ and $7.4 \mathrm{~K}$ ). In figure 7(a) the dashed red line depicts the superconducting transition according to the Boltzmann-type sigmoidal function:

$$
\rho_{\text {fit }}=\rho_{\text {high }}-\frac{\rho_{\text {high }}}{1+\mathrm{e}^{\left(T-T_{\mathrm{c}}\right) / \Delta T}},
$$

with $\rho_{\text {high }}$ being the normal state resistivity at $T=8 \mathrm{~K}$ and $\Delta T$ the slope factor. Here, $T_{\mathrm{c}}=7.39 \mathrm{~K}$ is calculated from the $\rho_{\mathrm{fit}}$-data (the dashed red line in figure $7(a)$ ) and taken as the temperature, where the resistivity drops by $50 \%$ with respect to the value of the normal state resistivity at $8 \mathrm{~K}$. Subtracting the received superconducting contribution from the experimental data results in a peak-like feature (the solid green line in figure $7(a)$ ). From this differential curve two characteristic temperatures are obtained. $T_{\mathrm{p} 1}(T)$ is defined by the intersection of the Boltzmann curve with the difference curve, and $T_{\mathrm{p} 2}(T)$ marks the position of the maximum of the extracted peak. With increasing magnetic fields the shoulder shifts to lower temperatures, until it renders into a separate peak, whose height increases with increasing fields (figure $7(b)$ ). In this case, $T_{\mathrm{p} 1}$ and $T_{\mathrm{p} 2}$ are marked as the local minimum and maximum of $\rho(T)$, respectively. The results of this investigations are summarized in the $H$ - $T$-phase diagram for $H_{\|}$in figure $9(a)$. It should be mentioned that the $H$-T-phase diagrams of the intercalated sample and the host substance in the highly dense vortex liquid phase are very similar, with exception of the upper critical field area [14].

The presence of a peak effect is also supported by AC-susceptibility measurements. In figures $8(a)$ and $(b)$ the imaginary and real part of the AC-susceptibility, $\chi_{V}^{\prime \prime}(T)$ and $\chi_{V}^{\prime}(T)$, are plotted for $\mathrm{NbSe}_{2}\left\{\mathrm{CoCp}_{2}\right\}_{0.26}$ in an applied magnetic DC-field of $2 \mathrm{~T}$ and 3T perpendicular to the layers. Similar to the resistivity measurements, a pronounced peak effect is determined from the AC-susceptibility data. For $\chi_{V}^{\prime}(T)$ a distinct peak develops, again characterized by the two temperatures $T_{\mathrm{p} 1}$ and $T_{\mathrm{p} 2}$, which mark the local minimum 

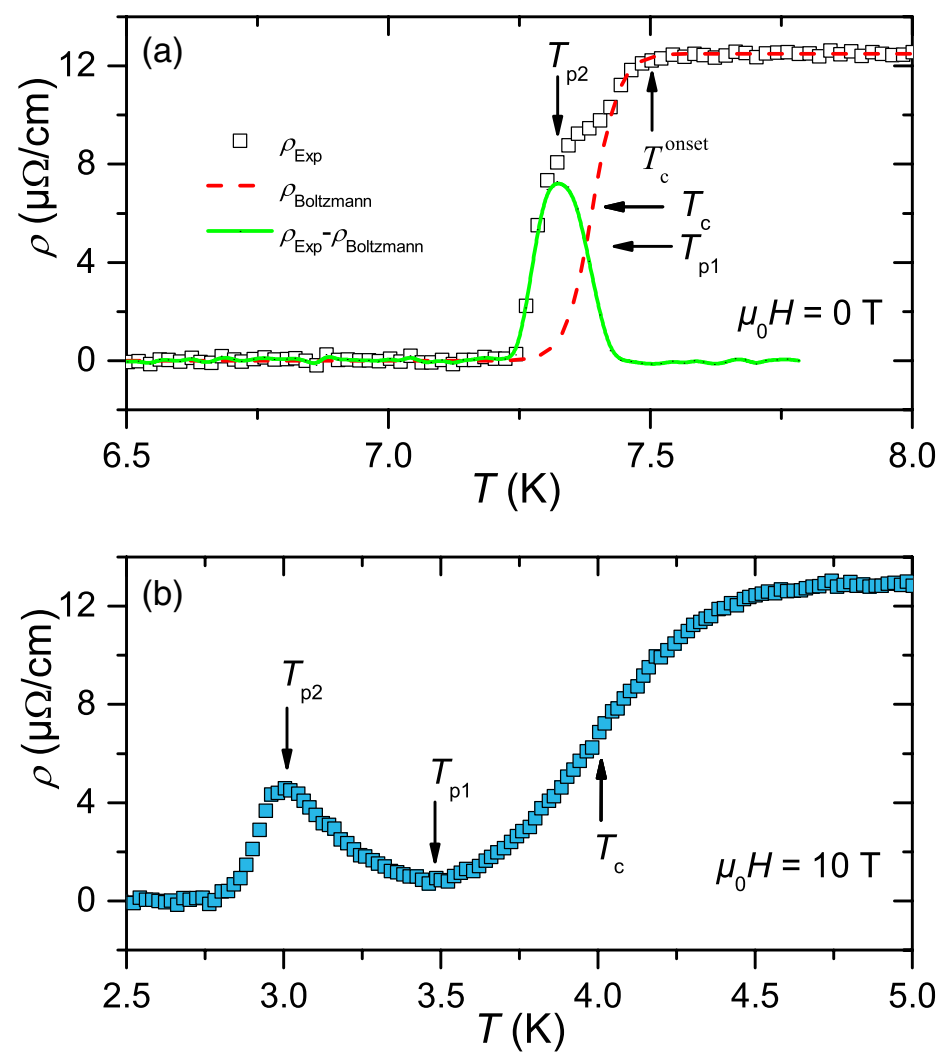

Figure 7. An enlarged presentation of the temperature dependent electrical resistivity in an applied magnetic field of $(a) 0 \mathrm{~T}$ and b) $10 \mathrm{~T}$ perpendicular to the layers. In $(a)$ the red dashed line represents the Boltzmann sigmoidal function adapted to the data, and the green line is the differential plot between the measured data and the adapted curve. For the definitions of the temperatures $T_{\mathrm{c}}^{\text {onset }}, T_{\mathrm{c}}, T_{\mathrm{p} 1}$ and $T_{\mathrm{p} 2}$ see text.

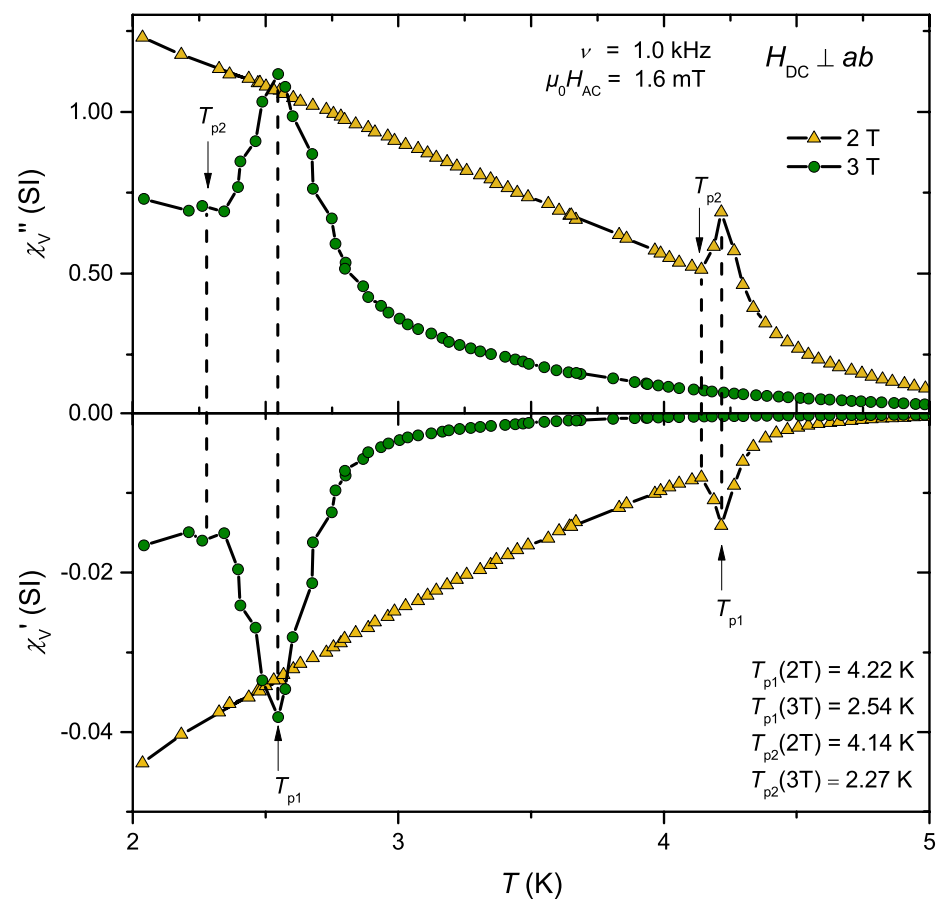

Figure 8. The temperature dependent AC-susceptibility $\left(v=1 \mathrm{kHz} ; \mu_{0} H_{\mathrm{AC}}=1.6 \mathrm{mT}\right)(a) \chi_{V}^{\prime \prime}$ and $(b) \chi_{V}^{\prime}$ of $\mathrm{NbSe}_{2}\left\{\mathrm{CoCp}_{2}\right\}_{0.26}$ in an applied DC-magnetic field of $2 \mathrm{~T}$ and $3 \mathrm{~T}$ perpendicular to the layers.

and the local maximum, respectively. However, in the cases of $\chi_{V}^{\prime \prime}(T)$ the situation is reversed (see figures $8(a)$ and $(b)$ ). The resulting temperature-dependent characteristic magnetic fields, $\mu_{0} H_{\mathrm{p} 1 \perp}(T)$ and $\mu_{0} H_{\mathrm{p} 2 \perp}(T)$, are summarized in the
$H$-T -phase diagram for $H_{\perp}$ in figure $9(b)$. In the case of $H_{\|}(T)$ (figure $\left.9(a)\right) \chi_{V}^{\prime}(T)$ and $\chi_{V}^{\prime \prime}(T)$ could only be analyzed up to $0.6 \mathrm{~T}$. Nevertheless, for this low field region the $H_{\|}(T)$ values of the characteristic temperatures, determined from 

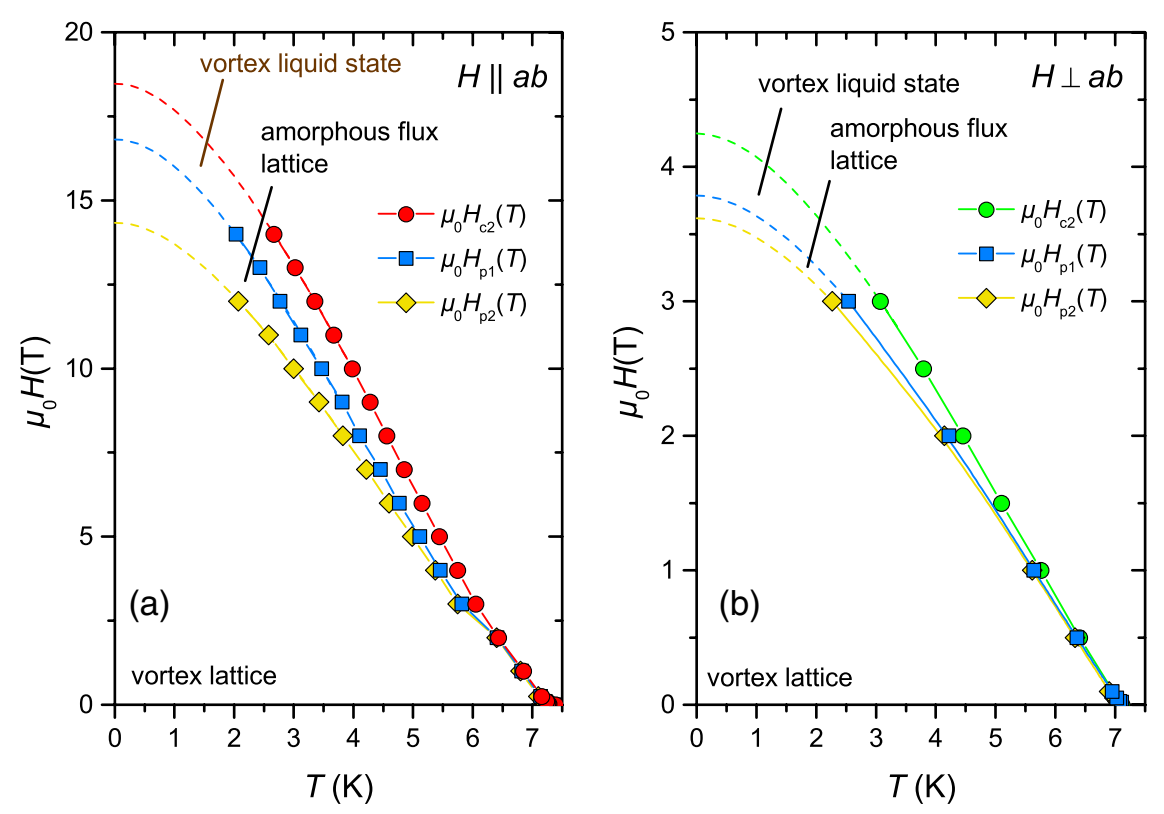

Figure 9. $H$-T -phase diagram of $\mathrm{NbSe}_{2}\left\{\mathrm{CoCp}_{2}\right\}_{0.26}$ in an applied magnetic field $(a)$ parallel and $(b)$ perpendicular to the layers. The phase boundaries for the vortex-liquid phase and the amorphous vortex lattice phase are determined in the case of $H \| a b$ from the resistivity measurements and for $H_{\perp} a b$ from the AC-susceptibility data.

the susceptibility data, nicely agree with the values from the resistivity measurements [14].

The phase diagrams in both directions (figures $9(a)$ and (b)) exhibit three different phases in the mixed state which broaden with increasing magnetic fields. Such behavior is also found in $2 \mathrm{H}-\mathrm{NbSe}_{2}$ for $H$ perpendicular to the layers [33, 45]. For the dilute vortex state in low magnetic fields, no phase separation up to $2 \mathrm{~T}$ for $H_{\|}$and $0.5 \mathrm{~T}$ for $H_{\perp}$ is observed. For the host sample a clear separation occurs in this region, which broadens with decreasing fields [46]. This different behavior in the dilute vortex region may be due to an unusual quantized flux penetration, as discussed in section 4.2.

In weakly pinned type-II superconductors, the vortex assemblage acts more or less as an elastic medium in a random pinning environment which can be activated thermally or driven by disorder. In both cases the shear forces between the single vortices get stronger than the pinning force. Intrinsic pinning centers are most likely in the large gaps between the superconducting layers; a scenario which is also discussed for HTC superconductors [47]. In the latter case, the mixed phase is not homogeneous but consists of different complex vortex phases [43]. In the simplest case the vortex lattice changes from a solid state, where the vortex array is fixed in an ordered Abrikosov- or in a slightly disordered Bragg phase, to an amorphous state (glass phase) and, finally, into a vortex liquid state, where the vortex flux motion is reversible. In comparison to the phase diagram of $2 \mathrm{H}-\mathrm{NbSe}_{2}$, the region between the $\mu_{0} H_{\mathrm{c} 2}$ and $\mu_{0} H_{\mathrm{p} 1}$ borderlines is related to the liquid state, followed by an amorphous state between the $\mu_{0} H_{\mathrm{p} 1}$ and $\mu_{0} H_{\mathrm{p} 2}$ lines. Due to the complex nature of vortex phases, a vast number of detailed studies have been performed in the case of high $T_{\mathrm{c}}$ as well as low $T_{\mathrm{c}}$ superconductors, see, e.g. $[10,33,48,49]$. To analyze the complicated vortex phase structure in $\mathrm{NbSe}_{2}\left\{\mathrm{CoCp}_{2}\right\}_{0.26}$, further investigations are warranted which also consider the subtle pinning behavior of this type-II superconductor in greater detail, as well as the presence of large van der Waals gaps and intercalated guest molecules which might act as pinning centers.

\section{Anisotropy and the specific heat}

The unusual temperature dependence of the electronic specific heat $\Delta C$, which is shown in figure $5(a)$ for the intercalated material $\mathrm{NbSe}_{2}\left\{\mathrm{CoCp}_{2}\right\}_{0.26}$, is also evidenced by the parent compound $2 \mathrm{H}-\mathrm{NbSe}_{2}$ and thus not controlled by the presence/absence of charge donation by the guest molecules. This feature has, however, raised an intense discussion on the role of the anisotropy of the material on $\Delta C(T)$ sequences. Kobayashi et al showed already in 1977 that the almost linear dependence of $\Delta C / T$ on $T^{2}$ can be explained by the anisotropy of the layered $2 \mathrm{H}-\mathrm{NbSe}_{2}$ [13]. They assumed an enhancement of the effective electron mass $m_{\perp}^{*}$ in the out-of-plane direction, as compared to the effective mass $m_{\|}^{*}$ in the planes, as well as a reduction in the superconducting energy gap perpendicular to the plane as an origin of the peculiar $\Delta C(T)$ behavior. The discovery of a similar, almost linear $T^{2}$-dependence of $\Delta C / T$ in the two-gap superconductor $\mathrm{MgB}_{2}$ [7], however, led to the supposition that in $2 \mathrm{H}-\mathrm{NbSe}_{2}$ the presence of two energy gaps with unequal magnitudes might also be the essential prerequisite for this $\Delta C(T)$ feature. Huang et al tested this hypothesis by fitting the specific heat obtained through various anisotropic gap models to their experimental data and compared these results with fits relying on a two-gap model $[29,50]$. These authors focused, however, mainly on the comparison of a two-gap model versus a superconducting (single) energy gap model with a six-fold symmetric in-plane anisotropy, which is supposed to reflect the symmetry of the crystal lattice. Their conclusion was that the two energy gap model performs better at describing the experimental data in the case of the selected benchmark systems. 

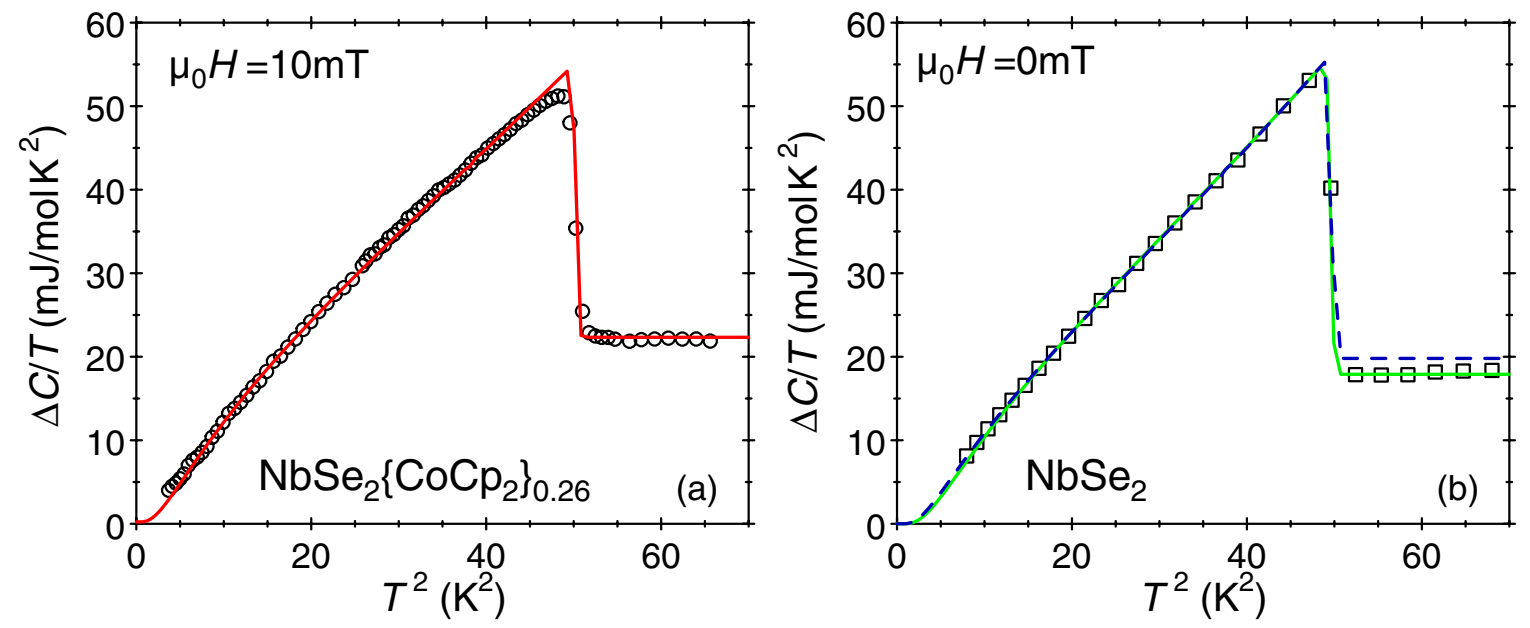

Figure 10. Fits (the solid lines) to the experimental data (the circles and squares) for $\Delta C / T$ as a function of $T^{2}$ for $(a)$ the intercalated $\mathrm{NbSe}_{2}\left\{\mathrm{CoCp}_{2}\right\}$ (data also shown in figures $5(a)$ and $(b)$ ) the parent compound $2 \mathrm{H}-\mathrm{NbSe}_{2}$, respectively. In $(a)$ the ratio $\Delta(T=0) / T_{\mathrm{c}}=1.76$ is fixed to the BCS value and an anistropy $v=m_{\|}^{*} / m_{\perp}^{*}=0.08$ is used. In $(b)$, the ratio $\Delta(T=0) / T_{\mathrm{c}}=2.09$ is adapted to reproduce the correct value for $\delta C / \gamma T_{\mathrm{c}}$ and an anisotropy $v=0.6$ is used (the solid green line). The dashed line shows $\Delta C / T$ for an isotropic system $(v=1)$, where $\Delta(T=0) / T_{\mathrm{c}}=1.99$ is adapted to reproduce $\Delta C / T$ best below $T_{\mathrm{c}}$.

Since our benchmark system allows a direct control of the sample's anisotropy factor via intercalation, we repeated the comparison of the performance of these different models to fit the $C(T)$ data in low dimensional superconductors. Our approach follows the ansatz of Kobayashi et al [13] and assumes an enhanced effective mass $m_{\perp}^{*}$ reflecting the system's anisotropy, whereas the gap function $\Delta$ is kept constant. The electronic specific heat of the superconducting state is given within the BCS theory [28] as:

$$
C=\frac{2 N_{0}}{T} \int \frac{\mathrm{d}^{2} \mathbf{k}}{4 \pi} \frac{\partial f_{\mathbf{k}}}{\partial E_{\mathbf{k}}}\left(E_{\mathbf{k}}^{2}-\frac{T}{2} \frac{\mathrm{d} \Delta^{2}(T)}{\mathrm{d} T}\right),
$$

where $f_{\mathbf{k}}=1 /\left(1+\mathrm{e}^{E_{\mathbf{k}} / k_{\mathrm{B}} T}\right)$ is the Fermi distribution function and $E_{\mathbf{k}}=\sqrt{\epsilon_{\mathbf{k}}^{2}+\Delta^{2}}$ is the quasi-particle energy. The temperature dependence of $\Delta(T)$ is derived from the selfconsistency condition $1 / V=\int \mathrm{d}^{2} \mathbf{k}\left(1-2 f_{\mathbf{k}}\right) / 2 E_{\mathbf{k}}$, where $V$ is the pairing interaction strength. The value of $V$ is chosen to give the measured critical temperature $T_{\mathrm{c}}$. The lattice anisotropy enters through the kinetic energy $\epsilon_{\mathbf{k}}=$ $\left(\hbar^{2} / 2\right)\left(\mathbf{k}_{\|}^{2} / m_{\|}^{*}+k_{\perp}^{2} / m_{\perp}^{*}\right)$, where $\mathbf{k}_{\|}$is the in-plane momentum and $k_{\perp}$ the out-of-plane momentum.

In order to find a model which reproduces the $\Delta C / T$ data best, it is important to notice that the ratio $\Delta(T=0) / T_{\mathrm{c}}$ has a fixed value within the BCS theory, which is $\approx 1.76$ for an isotropic gap function $\Delta$. Nevertheless, this ratio deviates somewhat from the BCS value in most superconductors, in particular, it is slightly enhanced in $2 \mathrm{H}-\mathrm{NbSe}_{2}$. A consequence of this enhancement is an increased specific heat jump $\delta C / \gamma T_{\mathrm{c}}$, for which the reported values vary somewhat (see table 1). The shape of the calculated curve $\Delta C / T$ depends strongly on the chosen value for the ratio $\Delta(T=0) / T_{\mathrm{c}}$ (see, e.g. [50]), wherefore this ratio is taken as a fitting parameter in $[13,29,50]$. In our measurements on $\mathrm{NbSe}_{2}\left\{\mathrm{CoCp}_{2}\right\}_{0.26}$, we find that the ratio $\delta C / \gamma T_{\mathrm{c}} \approx 1.43$ is in perfect agreement with the BCS result (figure 10(a)). The non-intercalated $2 \mathrm{H}-\mathrm{NbSe}_{2}$ (figure 10(b)), however, deviates from the BCS result with a ratio $\delta C / \gamma T_{\mathrm{c}} \approx 2.05$.

Because of the good agreement of $\mathrm{NbSe}_{2}\left\{\mathrm{CoCp}_{2}\right\}_{0.26}$ with the BCS result, we chose as a model ansatz for $\Delta C$ the pure BCS model with one isotropic gap and only the one fitting parameter $v=m_{\perp}^{*} / m_{\|}^{*}$. Varying $v$ does not alter the ratio $\delta C / \gamma T_{\mathrm{c}}$, but changes the curvature of $\Delta C / T$, which makes this model especially suitable for reproducing our measured data. Figure 10(a) presents our data for $\mathrm{NbSe}_{2}\left\{\mathrm{CoCp}_{2}\right\}_{0.26}(a)$ ) for $\mu_{0} H=10 \mathrm{mT}$ (which is almost identical to $\mu_{0} H=0$ ), and the calculated results (the solid red line) for $v=0.08$. While the low- and middle-temperature regime is remarkably well reproduced by our model, the measured $\Delta C / T$ is somewhat reduced close to $T_{\mathrm{c}}$. This reduction, which is also reported in [51], is not captured in the BCS description but is likely caused by a regime of enhanced superconducting fluctuations around $T_{\mathrm{c}}$, as can be expected in dimensionally reduced systems.

Figure 10(b) presents our measured results for the parent compound $2 \mathrm{H}-\mathrm{NbSe}_{2}$ in a zero magnetic field. Because of the enhanced ratio $\Delta C / \gamma T_{\mathrm{c}}$, this result is only reproducible if $\delta C / \gamma T_{\mathrm{c}}$ is used as a fitting parameter as well. The solid green line shows the calculated result with anisotropy $v=0.6$ and $\Delta(T=0) / T_{\mathrm{c}}=2.09$. Within this model, the data shown in $[13,29,30]$ are also well reproducible. Remarkably, $\Delta C / T$ is also ideally reproduced below $T_{\mathrm{c}}$ for an isotropic model $(v=1)$ if $\Delta(T=0) / T_{\mathrm{c}}$ is adapted only for $T<T_{\mathrm{c}}$, ignoring the height of the discontinuity in $\Delta C / T$. This is illustrated by the dashed line in figure $10(b)$. This means that if one allows for the presence of an additional mechanism (which is not captured in equation (6)) influencing $\delta C / \gamma T_{\mathrm{c}}$, then an isotropic single-gap model with an isotropic Fermi surface is sufficient to explain the $\Delta C / T$ data.

These observations allow the conclusion that both the intercalated and the non-intercalated material exhibit an $m_{\perp}^{*} / m_{\|}^{*}$ anisotropy, but while the non-intercalated material harbors an essentially three-dimensional electronic state $(v=$ 0.6 , the single $\mathrm{NbSe}_{2}$ layers are almost decoupled in the 
intercalated material $(v=0.08)$. Nevertheless, both materials exhibit a three-dimensional superconducting state, although the anisotropy is increased through intercalation (see table 1). A possibly anisotropic gap, as well as the presence of a second gap, may still add to the characteristic, almost linear $\Delta C / T\left(T^{2}\right)$ behavior of $\mathrm{NbSe}_{2}$. In view of the generally good agreement of our measurements with the predictions from the BCS theory and their reproducibility by a model ansatz using a single anisotropic Fermi surface, we believe, however, that the main effect on $\Delta C / T$ originates from the anisotropy in the effective masses $m_{\|}^{*}$ and $m_{\perp}^{*}$.

\section{Conclusion}

The layered superconductor $2 \mathrm{H}-\mathrm{NbSe}_{2}$ has been intercalated with the organometallic donor molecule cobaltocene to yield single-phase and crystalline $\mathrm{NbSe}_{2}\left\{\mathrm{CoCp}_{2}\right\}_{0.26}$ samples. A small increase in the superconducting transition temperature $T_{\mathrm{c}}^{\text {onset }}=7.5 \mathrm{~K}$ is observed upon intercalation, which represents the highest value of the intercalated or substituted $2 \mathrm{H}-\mathrm{NbSe}_{2}$ species reported so far. Structural characterization confirms that the lattice spacing perpendicular to the superconducting layers increases significantly upon intercalation and is twice as large as for the host lattice. This expansion alters the superconducting properties within the layers only marginally, but has a strong influence on the nature of the superconductivity perpendicular to the $\mathrm{NbSe}_{2}$ layers. The virtually decoupled arrangement of the $\mathrm{NbSe}_{2}$ layers in the intercalate increases the anisotropy factor of $\Gamma=H_{\mathrm{c} 2 \|} / H_{\mathrm{c} 2 \perp}=4.4$ by $34 \%$ relative to native $2 \mathrm{H}-\mathrm{NbSe}_{2}$. This observation is paralleled by an increase in the upper critical field $\mu_{0} H_{\mathrm{c} 2 \|}=18.5 \mathrm{~T}$ versus the parent compound $2 \mathrm{H}-\mathrm{NbSe}_{2}(14.1 \mathrm{~T})$. The fact that $\Gamma$ calculated from the $\xi$ ratio differs strongly from the corresponding values derived from the $\lambda$ - and $\kappa$-ratio provides another hint that the anisotropic GL theory fails to describe the incidence of superconductivity in $\mathrm{NbSe}_{2}\left\{\mathrm{CoCp}_{2}\right\}_{0.26}$. In addition, $\mathrm{NbSe}_{2}\left\{\mathrm{CoCp}_{2}\right\}_{0.26}$, as well as $2 \mathrm{H}-\mathrm{SnSe}{ }_{2}$, exhibits a reentrant peak effect in the magnetic fields parallel and perpendicular to the layers. This observation suggests the presence of complex vortex phases in the mixed state as a second contribution to the superconducting scenario.

Although the intercalated dichalcogenide $\mathrm{NbSe}_{2}$ $\{\mathrm{CoCp}\}_{0.26}$ exhibits a strongly enhanced anisotropy, surprisingly we found that the ratio $\delta C / \gamma T_{\mathrm{c}}=1.43$ is in good agreement with the prediction from the BCS theory. Based on the $\Delta C / T$ data fits, the superconducting scenario in both dichalcogenides, $\mathrm{NbSe}_{2}\left\{\mathrm{CoCp}_{2}\right\}_{0.26}$ and the parent compound $2 \mathrm{H}-\mathrm{NbSe}_{2}$, can be described by a one-gap BCS model with an anisotropic Fermi surface in contrast to earlier findings in the literature, which favored a two-gap scenario.

\section{Acknowledgments}

This work was supported by the Deutsche Forschungsgemeinschaft (project number SCHE487/12-1 and TRR 80).

\section{References}

[1] Klemm R A 2012 Layered Superconductors vol 1 (Oxford: Oxford University Press) chapter 8
[2] Scherer W et al 2010 Angew. Chem. Int. Edn 491578

[3] Scheidt E-W, Hauf C, Reiner F, Eickerling G and Scherer W 2011 J. Phys.: Conf. Ser. 273012083

[4] Nagamatsu J, Nakagawa N, Muranaka T, Zenitani Y and Akimitsu J 2001 Nature 41063

[5] Scheidt, E-W, Hathwar V R, Schmitz D, Dunbar A, Scherer W, Mayr F, Tsurkan V, Deisenhofer J and Loidl A 2012 Eur. Phys. J. B 85279

[6] Revolinsky E, Spiering G A and Beerntsen D J 1965 J. Phys. Chem. Solids 261029

[7] Bouquet F, Wang Y, Fisher R A, Hinks D G, Jorgensen J D, Junod A and Phillips N E 2001 Europhys. Lett. 56856

[8] Zehetmayer M and Weber H W 2010 Phys. Rev. B 82014524

[9] Meyer S F, Howard R E, Stewart G R, Acrivos J V and Geballe T H 1975 J. Chem. Phys. 624411

[10] Banerjee S S et al 1998 Europhys. Lett. 4491

[11] Harper J M E, Geballe T H and DiSalvo F J 1977 Phys. Rev. B 152943

[12] Rossnagel K, Seifarth O, Kipp L, Skibowski M, Voß D, Krüger P, Mazur A and Pollmann J 2001 Phys. Rev. B 64235119

[13] Kobayashi N, Noto K and Muto Y 1977 J. Low Temp. Phys. 27217

[14] Herzinger M 2013 Interkalationsverbindungen von $\mathrm{NbSe}_{2}$ und $\mathrm{SnSe}_{2}$ : Modellsysteme für niederdimensionale Supraleiter Dissertation Augsburg University

[15] Petrícek V, Dušek M and Palatinus L 2014 Z. Kristallogr. Cryst. Mater. 229345

[16] Dines M B 1975 Science 1881210

[17] Presnitz M 2014 Untersuchungen an Materialien mit niederdimensionalen Eigenschaften Dissertation, Augsburg University

[18] Toyota N, Nakatsuji H, Noto K, Hoshi A, Kobayashi N, Muto Y and Onodera Y 1976 J. Low Temp. Phys. 25485

[19] Ikebe M, Katagiri M, Noto K and Muto Y 1980 Physica B + C 99209

[20] Dalrymple B J and Prober D E 1984 J. Low Temp. Phys. 56545

[21] Naik I and Rastogi A K 2011 Pramana 76957

[22] Presnitz M, Herzinger M, Scheidt E-W, Scherer W, Baenitz M and Marz M 2012 Meas. Sci. Technol. 23085002

[23] Hillenius S J and Coleman R V 1979 Phys. Rev. B 204569

[24] Iavarone M, DiCapua R, Karapetrov G, Koshelev A E, Rosenmann D, Claus H, Malliakas C D, Kanatzidis M G, Nishizaki T and Kobayashi N 2008 Phys. Rev. B 78174518

[25] Scheidt E-W, Hucho C, Lüders K and Müller M 1989 Solid State Commun. 71505

[26] Denhoff M W and Gygax S 1982 Phys. Rev. B 254479

[27] Tinkham M 1996 Introduction to Superconductivity 2nd edn (New York: McGraw-Hill)

[28] Bardeen J, Cooper L N and Schrieffer J R 1957 Phys. Rev 1081175

[29] Huang C L, Lin J, Chang Y T, Sun C P, Shen H Y, Chou C C, Berger H, Lee T K and Yang H D 2007 Phys. Rev. B 76212504

[30] Jing Y, Lei S, Yue W, Zhi-Li X and Hai-Hu W 2008 Chin. Phys. B 172229

[31] Lin J Y, Hsieh Y S, Chareev D A, Vasiliev A N, Parsons Y and Yang H D 2011 Phys. Rev. B 84220507

[32] Lyard L et al 2002 Phys. Rev. B 66180502

[33] Tomy C V, Pal D, Banerjee S S, Ramakrishnan S, Grover A K, Bhattacharya S, Higgins M J, Balakrishnan B and Mck Paul D 2002 Pramana 58925

[34] Edwards J and Frindt R F 1971 J. Phys. Chem. Solids 322217

[35] Werthamer N R, Helfand E and Hohenberg P C 1966 Phys. Rev. 147295

[36] Klemm R A, Luther A and Beasley M R 1975 Phys. Rev. 12877 
[37] Angst M, Puzniak R, Wisniewski A, Jun J, Kazakov S M, Karpinski J, Roos J and Keller H 2002 Phys. Rev. Lett. 88167004

[38] Muto Y, Toyota N, Noto K and Hoshi A 1973 Phys. Lett. A 4599

[39] Ginzburg V L and Kirzhnits D A 1986 High Temperature Superconductivity (New York: Consultants Bureau)

[40] de Trey P, Gygax S and Jan J-P 1973 J. Low Temp. Phys. 11421

[41] Sanchez D, Junod A, Muller J, Berger H and Lévy F 1995 Physica B 204167

[42] Soto F, Berger H, Cabo L, Carballeira C, Mosqueira J, Pavuna D and Vidal F 2007 Phys. Rev. B 75094509

[43] Zhu X D, Lu J C, Sun Y P, Pi L, Qu Z, Ling L S, Yang Z R and Zhang Y H 2010 J. Phys.: Condens. Matter 22505704
[44] Mikitik G P and Brandt E H 2001 Phys. Rev. B 64184514

[45] Ghosh K et al 1996 Phys. Rev. Lett. 764600

[46] Ramakrishnan S et al 1996 Czech. J. Phys. 463105

[47] Tachiki M and Takahashi S 1989 Solid State Commun. 70291

[48] Thakur A D, Chandrasekhar Rao T V, Uji S, Trrashima T, Higgins M J, Ramakrishnan S and Grover A K 2006 J. Phys. Soc. Japan 75074718

[49] Thakur A D, Banerjee S S, Higgins M J, Ramakrishnan S and Grover A K 2006 Pramana J. Phys. 66159

[50] Huang C L, Lin J-Y, Sun C P, Lee T K, Kim J D, Choi E M, Lee S I and Yang H D 2006 Phys. Rev. B 73012502

[51] Kačmarčik J, Pribulová Z, Marcenat C, Klein T, Podière P, Cario L and Samuely P 2010 Phys. Rev. B 82014518 